\title{
Behavior of 3-hydrazino-6-aryl-1,2,4-triazin-5-one as a strong nucleophile towards active electrophilic compounds and their antibacterial evaluation
}

\author{
Abeer N. Al-Romaizan \\ Department of Chemistry, Faculty of Science, King Abdul Aziz University, Jeddah, KSA
}

\begin{abstract}
The behavior of 3-hydrazino-6-aryl-1,2,4-triazin-5-one towards the active electrophilic compounds in polar and/ or non-polar solvents and various times and temperatures, has been studied. $N$-[2-(3-(3/5-(4Nitrophenyl)-5/3-thioxo-1 $H$-1,2,4-triazol-1-yl)-5-oxo-1,2,4-triazin-6-yl)phenyl]pivalamides were obtained from the reaction of $N$-(2-(3-hydrazineyl-5-oxo-1,2,4-triazin-6-yl)phenyl)pivalamide with 4-nitrobenzoyl isothiocyanate in THF and/ or EtOH-piperidine respectively. Also, $N$-(2-(3-hydrazineyl-5-oxo-1,2,4-triazin-6yl)phenyl)pivalamide was shown a strong nucleophilic behavior by reaction with $N$-phenylthiourea to produce $N$-[2-(5-oxo-3-(2-(phenylcarbamothioyl)hydrazineyl)-1,2,4-triazin-6-yl)phenyl]pivalamide, which upon cyclization with diethyl carbonate produced $N$-(2-(5-oxo-3-(5-oxo-4-phenyl-3-thioxo-1,2,4-triazolidin-1-yl)1,2,4-triazin-6-yl)phenyl)pivalamide. Moreover, $N$-(2-(3-hydrazineyl-5-oxo-1,2,4-triazin-6-yl)phenyl)pivalamide studied its behavior by reaction with cyanoacetic acid, chloroacetonitrile, and/ or benzoyl carbonitrile to produce $N$-(2-(3-amino-4,8-dioxo-4H-[1,2,4]triazino[4,3-b][1,2,4]triazin-7-yl)phenyl)pivalamide, $N$-(2-(4-amino-8-oxo$2 H$-[1,2,4]triazino[4,3-b][1,2,4]triazin-7-yl)phenyl)pivalamide and $N$-(2-(4-imino-8-oxo-3-phenyl-4H-[1,2,4] triazino $[4,3-b][1,2,4]$ triazin-7-yl)phenyl)pivalamide. Structure of the products was established upon their elemental analysis and FT-IR, ${ }^{1} \mathrm{H} /{ }^{13} \mathrm{C}$ NMR, and MS. The new compounds were evaluated as antibacterial agents some Gram-positive and negative bacteria. Some compounds were showed the highest inhibition activity towards Pseudomonas aeruginosa, Bacillus subtilis, Bacillus cereus, and Sarcina lutea bacteria and lowest inhibitory activity against Escherichia coli bacteria.
\end{abstract}

Keywords: Behavior of 3-hydrazino-triazinone; Antibacterial; Electrophiles; Inhibition activity; Gram-positive and negative; Strong nucleophile

\section{Introduction}

Polyfunctionalized 1,2,4-triazine has significant vital probes properties, as biological, pharmacological, and agriculture applications, as anti-HIV ${ }^{1}$, anticancer ${ }^{2}$, antimicrobial ${ }^{3,4}$, photochemical probes for the inhibition of vitiligo, ${ }^{5}$ antibiotic resistances ${ }^{6}$. and anti-inflammatory ${ }^{6}$. On the other hand, phosphorusbearing 1,2,4-triazine mostly enhanced their physical, chemical, and biological properties. ${ }^{7,8}$ Also, 3-hydrazino-6-aryl-1,2,4-triazin-5-one used to produce heterobicyclic as anti-HIV and/or molluscicidal activity against some snails. ${ }^{7}$ As well as 3-thixio-1,2,4-traizin-5-one derivatives used as starting materials to obtain heteropolycyclic nitrogen systems as antioxidant and anti-inflammatory ?. $o$-Diamines as hydrazino moiety are very active substrates for the building of various heterobicyclic nitrogen systems ${ }^{9,10}$. Abdel-Rahman et al. ${ }^{11}$, have been investigated the behavior of hydrazino-groups towards various bi-electrophilic compounds. Based on these observations, and as a part of our continuing work in these areas ${ }^{12,13}$, the present work describes other attempts for the behavior of 3-hydrazino-1,2,4triazinone towards some activated electrophilic compounds in different medium and conditions in view of their bactericidal effects.

\section{Results and Discussion}

$N$-[2-(3-Hydrazino-5-oxo-2,5-dihydro-1,2,4-triazin6-yl)phenyl]pivalamide (3) as a starting material, was obtained from acylated 6-(2 aminophenyl)-3-thioxo1,2,4-triazin-5(4H)one (1) ${ }^{14}$ with $t$-butoyl chloride in warming DMF, followed by hydrazinolysis in $\mathrm{EtOH}$ Scheme 1. 


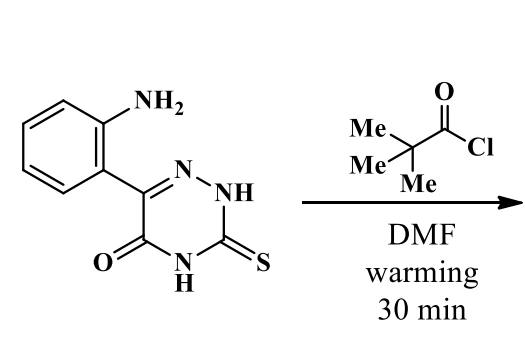

1<smiles>CC(C)(C)C(=O)Nc1ccccc1-c1n[nH]c(=S)[nH]c1=O</smiles>

2

$\mathrm{N} 2 \mathrm{H} 4 \cdot \mathrm{H} 2 \mathrm{O}$
$\mathrm{EtOH}$
reflux
$12 \mathrm{~h}$<smiles>CC(C)(C)C(=O)Nc1ccccc1-c1n[nH]c(NN)nc1=O</smiles>

3

Scheme 1. Synthesis of compounds $\mathbf{2}$ and $\mathbf{3}$

The main aim of this work is a study of the behavior of strong bi-nucleophilic as hydrazine groups towards activated poly electrophilic centers in polar and nonpolar solvents. Thus, refluxing of $N$-[2-(3hydrazino-5-oxo-2,5-dihydro-1,2,4-triazin-6-yl) phenyl] pivalamide (3) with 4-nitrobenzoyl isothiocyanate in THF was afforded $N$-[2-(3-(5-(4- nitrophenyl)-3-thioxo-2,3-dihydro- $1 H$-1,2,4-triazol1-yl)-5-oxo-2,5 -dihydro-1,2,4- triazin-6-yl)phenyl] pivalamide (4), while that reaction in EtOH/ drops of piperidine, produced $N$-[2-(3-(3-(4-nitrophenyl)-5thioxo-4,5-dihydro-1H-1,2,4-triazol-1-yl)-5-oxo-2,5 -dihydro-1,2,4-triazin-6-yl)phenyl]pivalamide

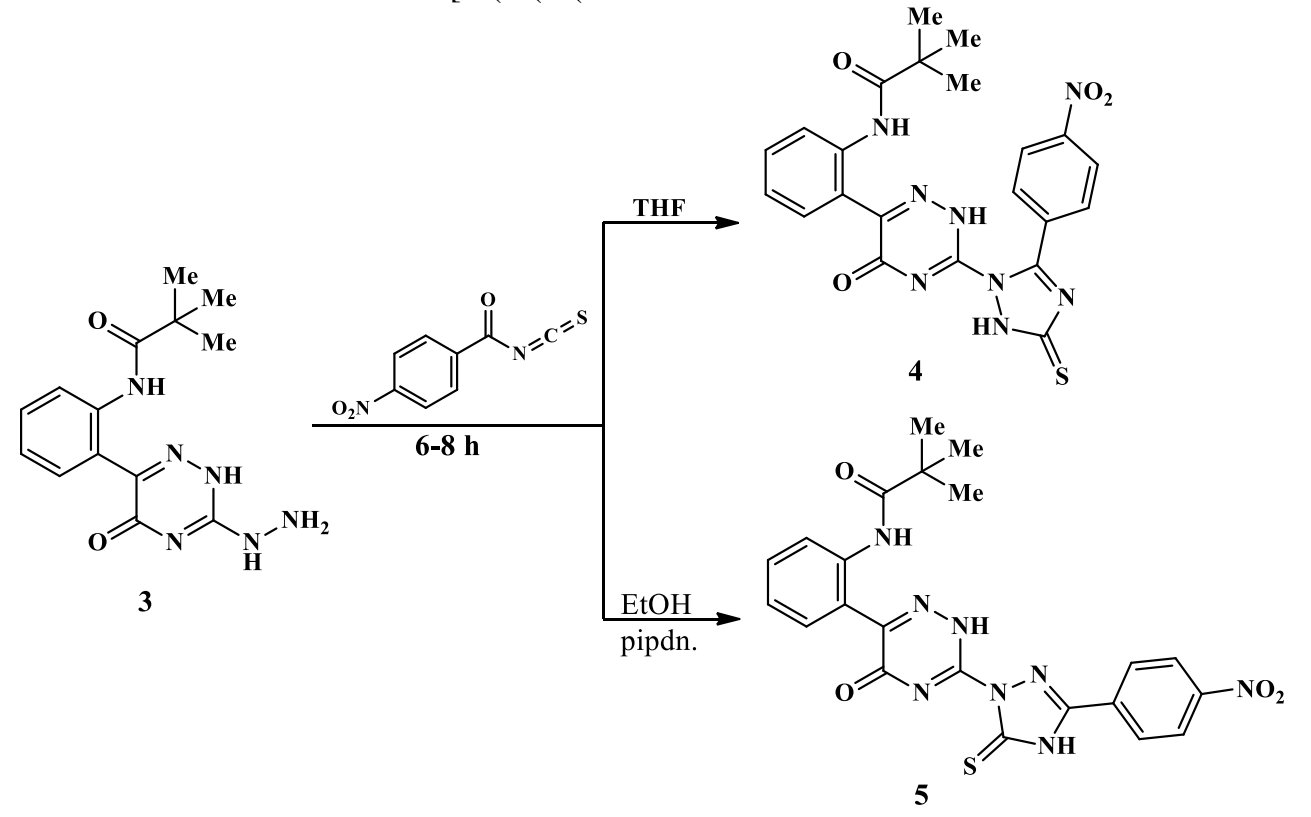

Scheme 2. Synthesis of compounds $\mathbf{4}$ and $\mathbf{5}$

Reaction of compound $\mathbf{3}$ took place via attacking a strong $\mathrm{NH}_{2}$ of hydrazino group on the carbon of $\mathrm{N}=\mathrm{C}=\mathrm{S}$ group of 4-nitrobenzoyl isothiocyanate in nonpolar solvent (THF), followed by elimination one mol of $\mathrm{H}_{2} \mathrm{O}$ to produce compound 4 , while in a polar solvent (EtOH-piperidine), the reaction took another way by attacking a strong $\mathrm{NH}_{2}$ of hydrazino group on the carbon of $\mathrm{O}=\mathrm{C}-\mathrm{N}$ group of 4-nitrobenzoyl isothiocyanate followed by elimination one mol of $\mathrm{H}_{2} \mathrm{O}$ (Fig. 1). 


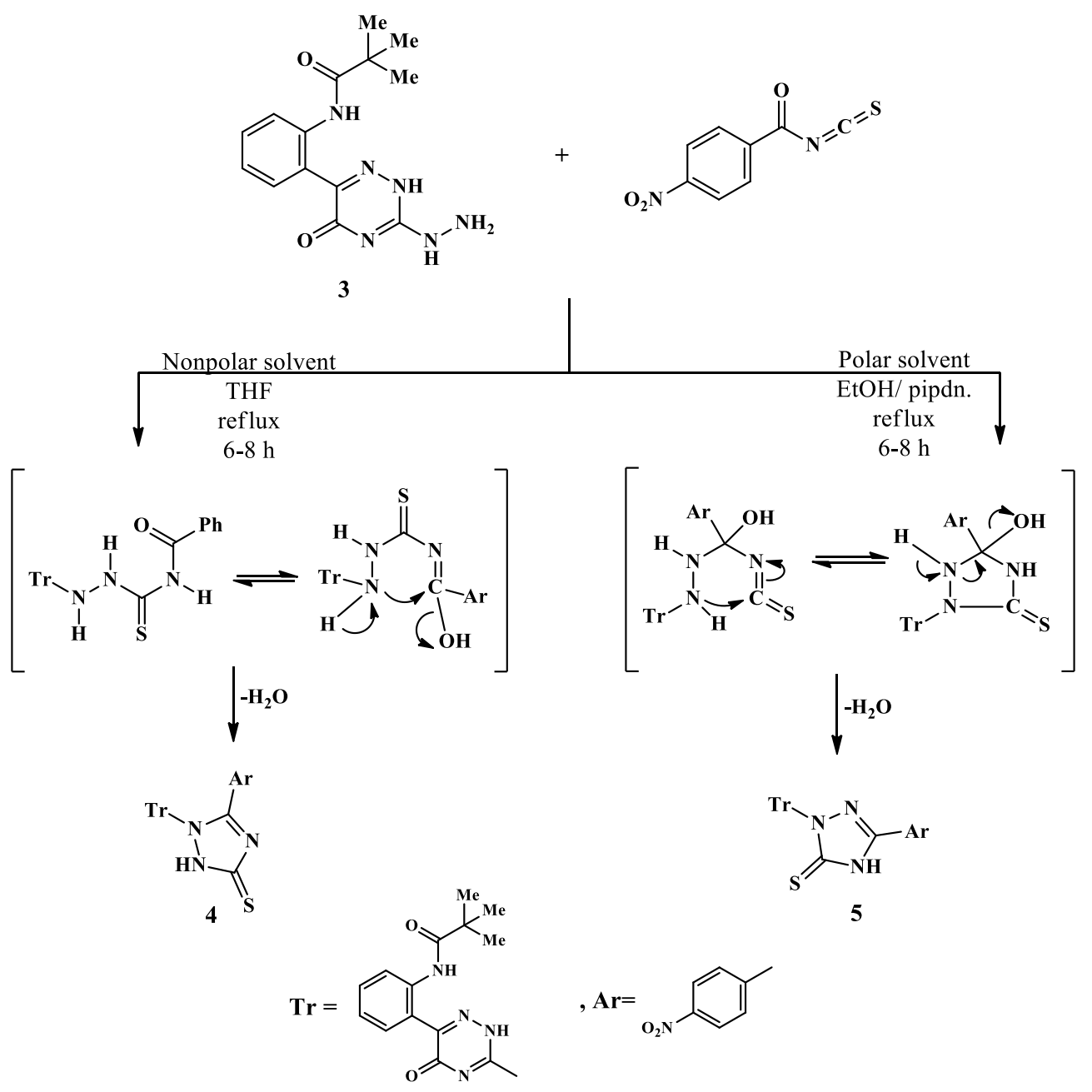

Figure 1. Formation of compounds 4 and 5 from 3

The isomeric structures $\mathbf{4} \& \mathbf{5}$ have a different only in their melting points and/or mass fragmentation pattern

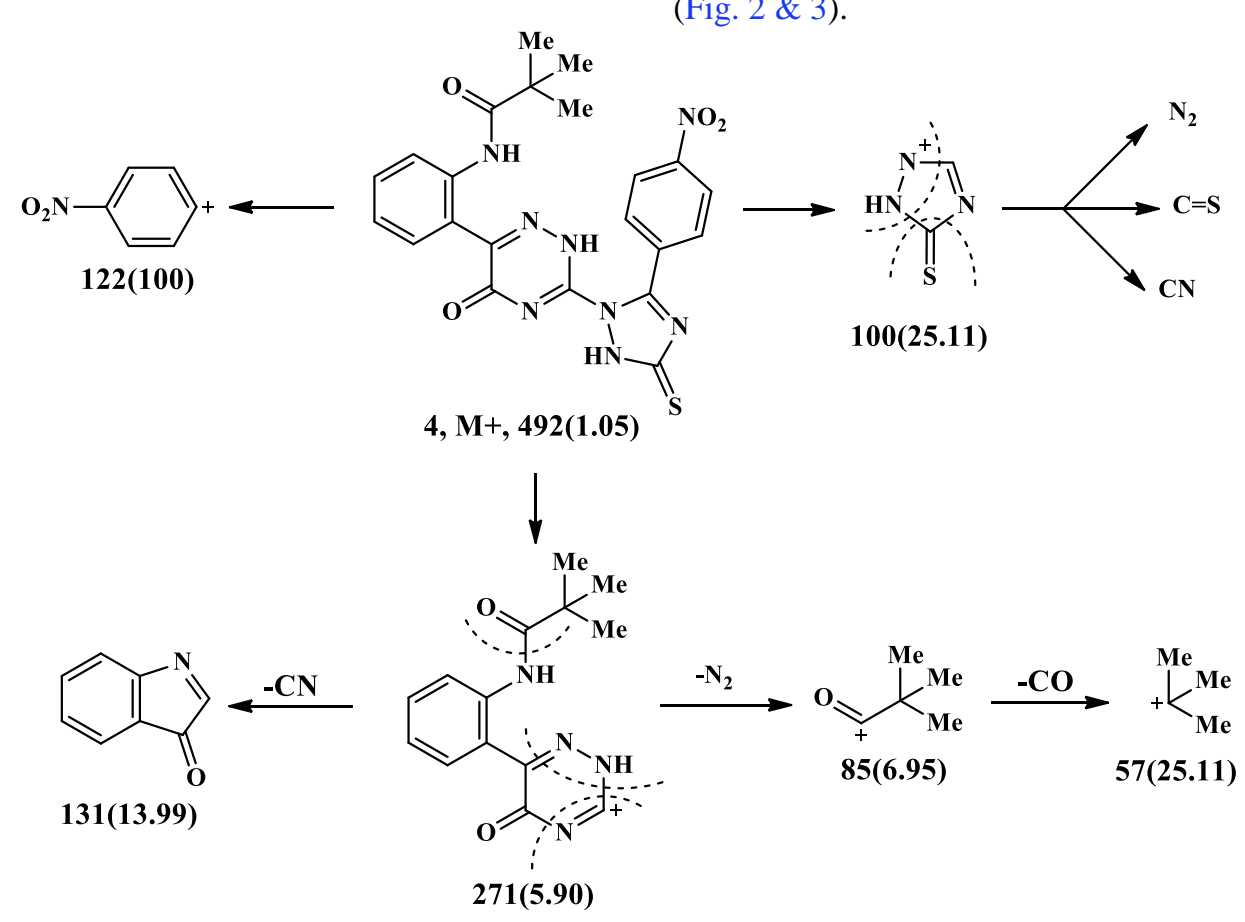

Figure 2. Mass fragmentation of compound 4 


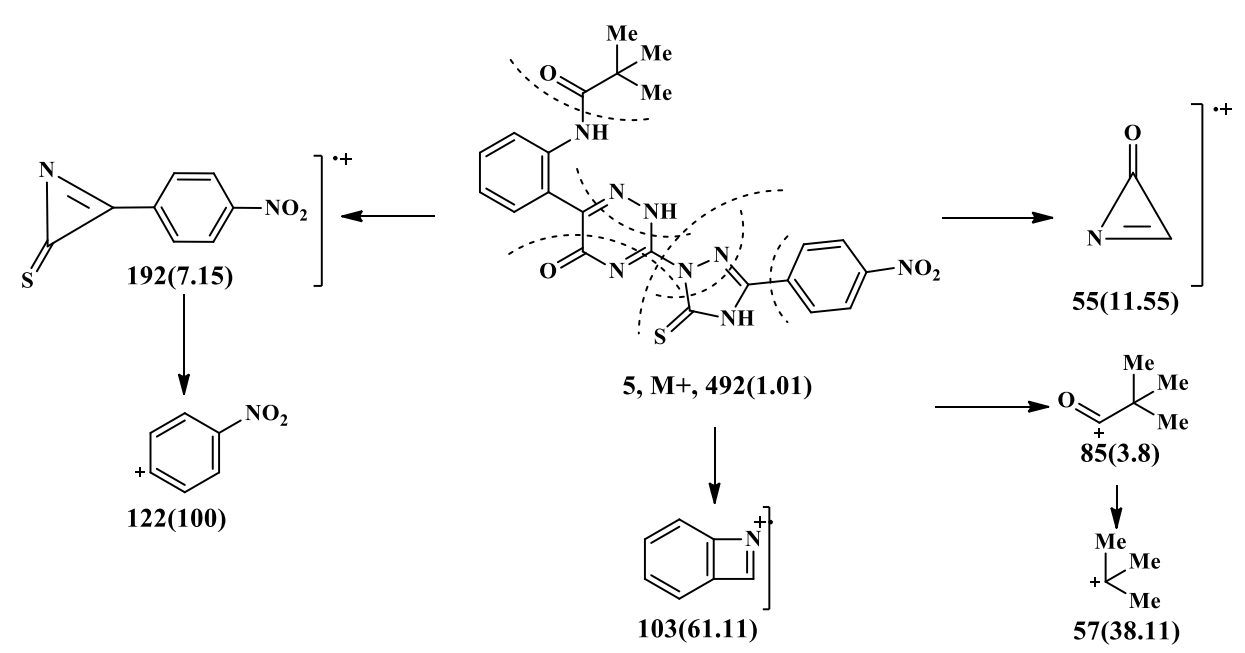

Figure 3. Mass fragmentation of compound 5

Formation of compound $\mathbf{7}$ may take place via a nucleophilic attack of $\mathrm{NH}_{2}$ of hydrazino moiety to a<smiles>CC(C)(C)C(=O)Nc1ccccc1-c1n[nH]c(NNCC2CC2)nc1=O</smiles>

3<smiles>CC(C)(C)C(=O)Nc1ccccc1-c1n[nH]c(-n2[nH]c(=N)n(-c3ccccc3)c2=O)nc1=O</smiles>

more electrophilic position $(\mathrm{C}=\mathrm{S})$ with the elimination of two mol of EtOH (Fig. 4).

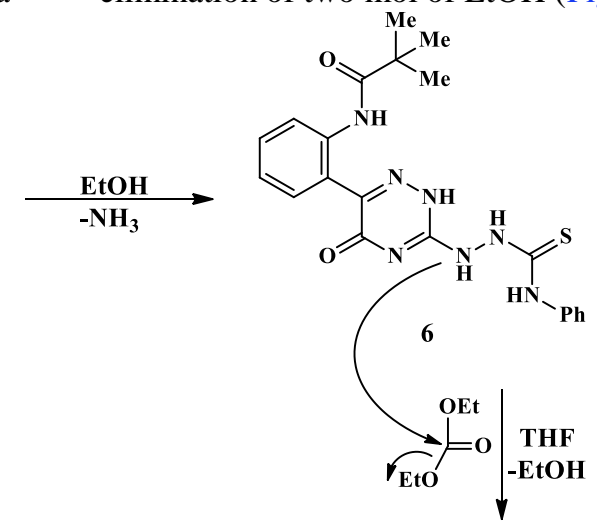<smiles>C1CCCCCCCCCC1</smiles>

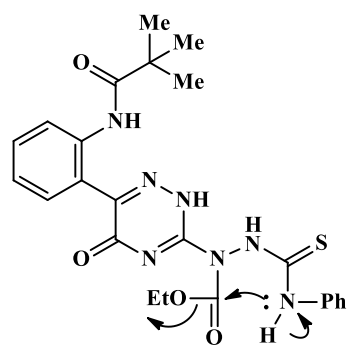

Figure 4. Formation of compound 7 from 3

It is known that thiourea derivatives are as amides, so the amino group is easily removable. Thus, thiourea is considered weak bi-electrophilic agents. Based upon this fact, refluxing of $N$-[2-(3-hydrazino-5-oxo-2,5dihydro-1,2,4-triazin-6-yl) phenyl]pivalamide (3) with $N$-phenylthiourea in abs. EtOH was yielded $N$-[2-(5-oxo-3-(2-(phenyl carbamothioyl)hydrazino)- 2,5-dihydro-1,2,4-triazin -6-yl)phenyl]pivalamide (6), which upon full heterocyclization by refluxing with diethyl carbonate in THF, led to the direct formation of $\mathrm{N}$-[2-(5-oxo-3-(5-oxo-4-phenyl-3thioxo-1,2,4-triazolidin-1-yl)-2,5-dihydro-1,2,4triazin-6-yl) phenyl]pivalamide (7) Scheme 3.<smiles>CC(C)(C)C(=O)Nc1ccccc1-c1n[nH]c(NN)nc1=O</smiles>

3

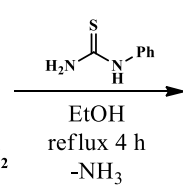

.<smiles>CC(C)(C)C(=O)Nc1ccccc1-c1n[nH]c(NNC(=S)Nc2ccccc2)nc1=O</smiles>

6

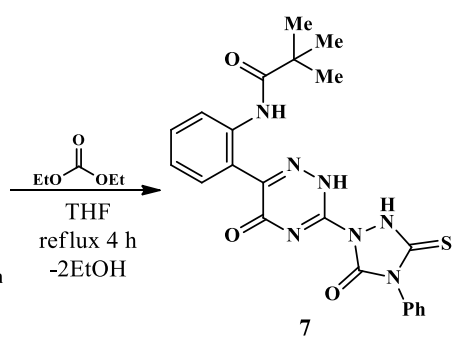

Scheme 3. Synthesis of compounds 6 and 7 
Moreover, the interaction between compound $\mathbf{3}$ as a strong nucleophile and cyanoacetic acid as bi-electrophile in ethanol with few drops of piperidine as a catalyst, yielded ${ }^{11} N$-(2-(3-amino-4,8-dioxo-1,8dihydro-4H-[1,2,4]triazino[4,3-b][1,2,4]triazin-7- yl)phenyl)pivalamide (8), while treatment with 2-chloroacetonitrile in DMF, afforded ${ }^{12} \mathrm{~N}$-(2-(4amino-8-oxo-1,8-dihydro- $2 \mathrm{H}$-[1,2,4]triazino[4,3b] $[1,2,4]$ triazin-7-yl)phenyl)pivalamide(9) respectively Scheme 4.<smiles>CC(C)(C)C(=O)Nc1ccccc1-c1nn2c(=O)c(N)n[nH]c2nc1=O</smiles>

8

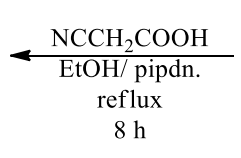

$8 \mathrm{~h}$<smiles></smiles>

3

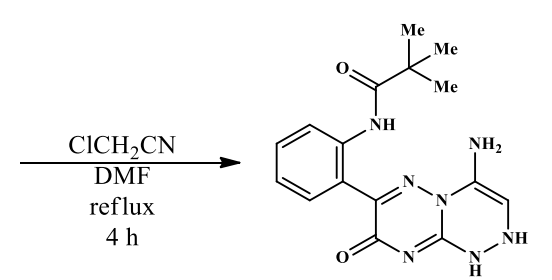

9

Scheme 4. Synthesis of compounds 8 and 9

Formation of both compounds $\mathbf{8}$ and $\mathbf{9}$ may be shown in (Fig. 5 \& 6).

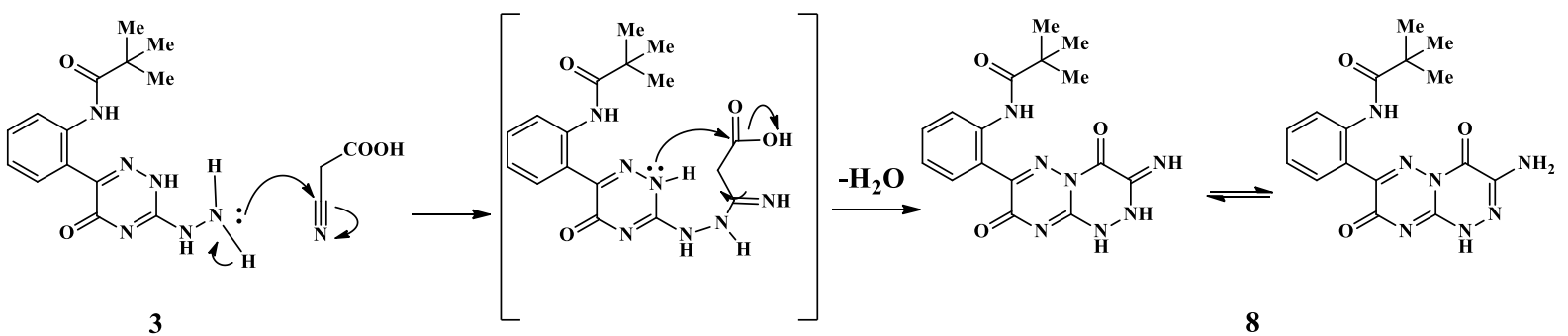

Figure 5. Formation of compound $\mathbf{8}$ from $\mathbf{3}$

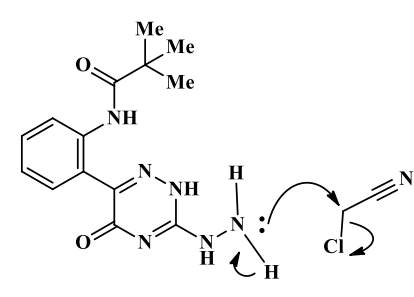

3

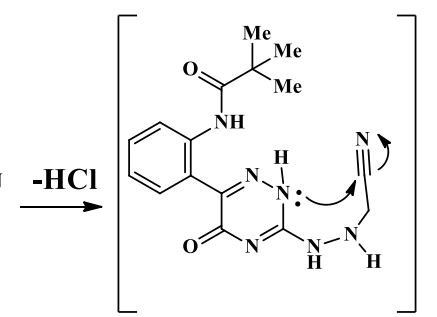

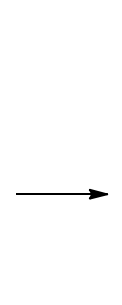<smiles>CC(C)(C)C(=O)N=C1N=C2C(=N)CNNC2=NC1=O</smiles><smiles></smiles>

9

Figure 6. Formation of compound 9 from 3

Abdel-Rahman et al. ${ }^{11}$ studied the reactivity of strong nucleophile reagents towards active carbonitriles as electrophile reagents. Similarly, treatment of 3-hydrazino-1,2,4-triazinone $\mathbf{3}$ with benzoyl carbonitrile in ethanol with drops of piperidine, yielded $N$-(2-(4-imino-8-oxo-3-phenyl-1,8-dihydro$4 H$-[1,2,4]triazino[4,3-b][1,2,4]triazin-7yl)phenyl)pivalamide (10) Scheme 5.<smiles>CC(C)(C)C(=O)Nc1ccccc1-c1n[nH]c(NN)nc1=O</smiles>

3

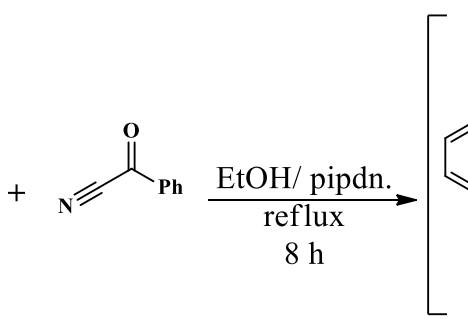

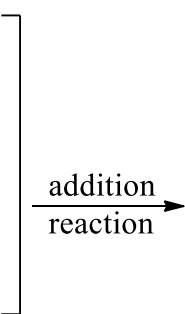<smiles>CC(C)(C)C(=O)Nc1ccccc1-c1nn2c(=N)c(-c3ccccc3)n[nH]c2nc1=O</smiles>

10

Scheme 5. Synthesis of compound $\mathbf{1 0}$ from $\mathbf{3}$

Former structures of new compounds obtained were deduced from their elemental analysis and spectral measurements. FT-IR absorption spectra of all compounds were showed $\bar{v}$ at $3300,3250,3150 \mathrm{~cm}^{-}$ 1 for $\mathrm{NH}$ functional groups, with $\bar{v}$ at 1670, 1660, $\& 1620,1600 \sim 1570, \& 1200 \sim 1188 \mathrm{~cm}^{-1}$ attribute to $\mathrm{C}=\mathrm{O}, \quad \mathrm{C}=\mathrm{N}$, and $\mathrm{C}=\mathrm{S}$ respectively. Only, the compound $\mathbf{4}$ and $\mathbf{5}$ were recorded $\bar{v}$ at 1530 \&
$1350 \mathrm{~cm}^{-1}$ for asymmetric and symmetric $\mathrm{NO}_{2}$. ${ }^{1} \mathrm{H}$ NMR spectra of all new compounds were recorded resonated signals at $\delta 12.11,10.55$, and $8.55 \mathrm{ppm}$ for exo NHCO, endo NHCO, and NHCS protons with $t$ butyl protons at $\delta 1.2,1.11$, and $0.99 \mathrm{ppm}$, besides the aromatic protons at $\delta 7.90-6.99 \mathrm{ppm}$. 
Moreover, ${ }^{13} \mathrm{C}$ NMR spectra of the targets were showed mainly $\delta$ at $188-180,168-152$, and $142 \mathrm{ppm}$ attribute to $\mathrm{C}=\mathrm{S}, \mathrm{C}=\mathrm{O}, \& \mathrm{C}=\mathrm{N}$ carbons, with aromatic carbons at $\delta$ at 132-126 ppm. The two isomers 4 and $\mathbf{5}$ have differences in melting points and mass spectra. So, mass fragmentation pattern gives us a good indication about these structures, compounds $\mathbf{4}$ and $\mathbf{5}$ were showed the molecular ion peak at $\mathrm{m} / \mathrm{z} 492$ with the base peak at $m / z 122$ (Fig. $2 \& 3$ ).

The former structures of compounds 6 and 7 have been confirmed from that FT-IR spectra were showed $\bar{v}$ at $1200-1180 \mathrm{~cm}^{-1}$ for $\mathrm{C}=\mathrm{S}$ functional group, while that of compounds 8 and $\mathbf{9}$ recorded $\bar{v}$ at 3200-3100 and $1640-1630 \mathrm{~cm}^{-1}$ for $\mathrm{NH}_{2}$ group. All compounds 8-10 showed a lacks $C N$ group, which confirms the addition reactions have happened. ${ }^{1} \mathrm{H}$ NMR spectra of compounds 8 and 9 recorded at 3.5 ppm for $\mathrm{NH}_{2}$ protons while that of compound $\mathbf{1 0}$ showed at $5.5 \mathrm{ppm}$ for $=\mathrm{NH}$ proton.

Finally, the synthesized compounds were exhibited antibacterial activity, thus, from the obtained results in Table 1, we can be concluded that: the compounds $\mathbf{4}, \mathbf{5}, \& \mathbf{7}$ are observed high inhibition activity towards $P$. aeruginosa, B. subtilis, $B$. cereus, and $S$. lutea bacteria and lowest inhibitory activity against $E$. coli bacteria. The MIC study of compounds $\mathbf{4}, \mathbf{5}$, and $\mathbf{7}$ shown in Table 2.

QSAR study showed that the higher activities of compounds 4, 5, \& 7 might be due to the presence of thioxo-1,2,4-triazole moiety as well as 4-nitrophenol groups which as bactericidal agents. Moreover, the presence of 6-aryl-1,2,4-triazin-5-one nucleus enhanced that activity. Also, compound $\mathbf{4}$ had a bioconjugated system between the two heterocyclic 1,2,4-triazole and 1,2,4-triazine nucleus.

In comparison between the activity of compounds, we can be concluded that 3-thioxo-1,2,4-triazole moiety gave a high activity than 5-thioxo-1,2,4-triazole moiety, which may be the presence of cyclic NCSN part within the bio-conjugated systems.

\section{Conclusion}

The behavior of 3-hydrazino-1,2,4-triazin-5-one as a strong nucleophile towards the active electrophilic compounds, in different media, has been investigated. Where the primary amino group firstly attacked the more electropositive atoms. $\mathrm{N}$-(2-(3-Hydrazineyl-5oxo-2,5-dihydro-1,2,4-triazin-6-yl)phenyl)pival-

amide was given tow reaction ways in polar and nonpolar solvents. All heterobicyclic systems obtained, were exhibited as in vitro antibacterial activity, especially Gram-positive bacteria, in comparing with Tetracycline as an antibiotic standard. Some compounds were showed a good result.

\section{Experimental}

All chemicals were purchased from Merck and BDH and used without any further purifications. The melting points were recorded on Stuart scientific SMP30 (Bibby, UK) melting point apparatus and reported as uncorrected. A Perkin Elmer model RXI-FT-IR 55,529 $\mathrm{cm}^{-1}$ was used for recording the FT-IR spectra. A Brucker advance DPX $400 \mathrm{MHz}$ using TMS as an internal standard was used for recording the ${ }^{1} \mathrm{H}$ and ${ }^{13} \mathrm{C}$ NMR spectra in deuterated DMSO $(\delta$ in $\mathrm{ppm})$ as a solvent. AGC-MS-QP 1000 ex-model was used for recording the mass spectra. Elemental microanalysis was performed on a Perkin-Elmer CHN-2400 analyzer. All reactions were monitored by TLC, using silica gel coated Al plates with fluorescent indicator F254. 4Nitrobenzoyl isothiocyanate was obtained from refluxing 4-nitrobenzoyl chloride with ammonium thiocyanate in dry acetone ${ }^{11}$, and 6-(2-aminophenyl)3-thioxo-1,2,4-triazin-5(4H) one (1) also obtained from refluxing isatin with thiosemicarbazide in $a q$. $\mathrm{NaOH}^{14}$, according to the reported methods.

Table 1. The in vitro antibacterial activities of compounds 4-8.

\begin{tabular}{|c|c|c|c|c|c|c|c|c|}
\hline \multirow{3}{*}{ Compound. } & \multicolumn{8}{|c|}{ Bacteria branch*/ Inhibition Zone $(\mathrm{Hz})^{* * *}$} \\
\hline & \multicolumn{4}{|c|}{ (+ve)-Bacteria } & \multicolumn{4}{|c|}{ (-ve)-Bacteria } \\
\hline & B. $s$. & B. $c$. & S. $l$. & M. $l$. & P. $a$. & E. c. & A. $j$. & X. o. \\
\hline 4 & 25 & 20 & 12 & 18 & 17 & 12 & 16 & 11 \\
\hline 5 & 18 & 17 & 11 & 14 & 15 & 10 & 11 & 10 \\
\hline 6 & 15 & 15 & 10 & 16 & 14 & 9 & 10 & 8 \\
\hline 7 & 20 & 17 & 11 & 15 & 16 & 11 & 16 & 11 \\
\hline 8 & 18 & 17 & 10 & 17 & 14 & 9 & 12 & 9 \\
\hline Tetracycline (Control) & 15 & 15 & 10 & 20 & 15 & 10 & 15 & 10 \\
\hline
\end{tabular}

*: B. s.: Bacillus subtilis, B. c.: Bacillus cereus, S. l.: Sarcina lutea, M. l.: Micrococcus luteus, P. a.: Pseudomonas aeruginosa, E. c.: Escherichia coli, A. j.: Acinetobacter johnsonii, X. o.: Xanthomonas oryzae. **: $50 \%$, used as a selective concentration. 
Table 2. The MCI study of activated compounds towards the selected microorganisms.

\begin{tabular}{|c|c|c|c|c|c|c|c|c|c|}
\hline \multirow[b]{3}{*}{ Organism* } & \multicolumn{9}{|c|}{ Compound } \\
\hline & \multicolumn{3}{|c|}{4} & \multicolumn{3}{|c|}{5} & \multicolumn{3}{|c|}{7} \\
\hline & $50 \%$ & $30 \%$ & $10 \%$ & $50 \%$ & $30 \%$ & $10 \%$ & $50 \%$ & $30 \%$ & $10 \%$ \\
\hline$P . a$. & 17 & 12 & 11 & 15 & 11 & 8 & 16 & 12 & 8 \\
\hline B. $s$. & 25 & 18 & 10 & 18 & 16 & 12 & 20 & 13 & 10 \\
\hline B. $c$. & 20 & 14 & 9 & 17 & 12 & 9 & 17 & 15 & 12 \\
\hline
\end{tabular}

*: P. a.: Pseudomonas aeruginosa, B. s.: Bacillus subtilis, B. c.: Bacillus cereus.

\section{N-[2-(5-Oxo-3-thioxo-2,3,4,5-tetrahydro-1,2,4-} triazin-6-yl) phenyl] pivalamide (2)

Compound 1 (17.5 g, $79.54 \mathrm{mmol})$ and $t$-butoyl chloride $(9.54 \mathrm{~g}, 79.54 \mathrm{mmol})$ in DMF $(150 \mathrm{ml})$ were warmed for $30 \mathrm{~min}$. The reaction mixture was cooled to the room temperature then poured onto ice. The solid obtained was filtered off and crystallized from $\mathrm{EtOH}$ to give $\mathbf{2}$ as

yellowish crystals. Yield 18.37 g, 76 \%, m.p: 344-345 ${ }^{\circ} \mathrm{C}$.

FT-IR (ATR, $\left.\bar{v}, \mathrm{~cm}^{-1}\right)$ : 3300, 3180(2NH), 3050(ArH), 2960, 2880(aliphatic CH), 1670(C=O), 1650(CONH), $1580(\mathrm{C}=\mathrm{N}), 1330($ cyclic NCSN), $1188(\mathrm{C}=\mathrm{S}), 880$, 810(aromatic ring).

${ }^{1} \mathrm{H}$ NMR (400 MHz, DMSO- $\left.d_{6}\right) \delta(\mathrm{ppm}): 11.55(\mathrm{~s}, 1 \mathrm{H}$, $\mathrm{NH}), 11.02(\mathrm{~s}, 1 \mathrm{H}, \mathrm{NH}), 10.85(\mathrm{~s}, 1 \mathrm{H}, \mathrm{NHCO}), 7.70-$ 7.42(m, 4H, aromatic), 1.90, 1.88, 1.21(each s, 9H, $3 \mathrm{CH}_{3}$ ).

${ }^{13} \mathrm{C}$ NMR (100 MHz, DMSO- $\left.d_{6}\right) \delta(\mathrm{ppm}): 188(\mathrm{C}=\mathrm{S})$, 162, $\quad 154(\mathrm{C}=\mathrm{O}), \quad 141(\mathrm{C}=\mathrm{N}), \quad 132-128$ (aromatic carbons), $119,116(\mathrm{C}=\mathrm{C}$ of 1,2,4-trizinone), 19.10, 18.88, and 18.79 (aliphatic carbons).

Calculated, $\mathrm{C}_{14} \mathrm{H}_{16} \mathrm{~N}_{4} \mathrm{O}_{2} \mathrm{~S}\left(\mathrm{M}^{+} 305\right)$, \%: C, 55.25; H, 5.30; N, 18.41; S, 10.53. Found, \%: C, 55.11; H, 5.19; N, 18.32; S, 10.44 .

\section{N-[2-(3-Hydrazino-5-oxo-2,5-dihydro-1,2,4-triazin- 6-yl)phenyl]pivalamide (3)}

A mixture of compound 2 (17.00 g, $55.92 \mathrm{mmol})$ and hydrazine hydrate $(25 \mathrm{ml})$ in ethanol $(150 \mathrm{ml})$ was heated under reflux for $12 \mathrm{~h}$. The reaction mixture was cooled to the room temperature then poured onto ice. The yielded solid, was filtered off and crystallized from EtOH to give $\mathbf{3}$ as ball

yellow crystals. Yield 11.99 g, $71 \%$, m.p: $359-360^{\circ} \mathrm{C}$. FT-IR (ATR, $\left.\bar{v}, \mathrm{~cm}^{-1}\right)$ : 3300, 3180, 3090(NH, $\left.\mathrm{NH}_{2}\right)$, 3060(ArH), 2980, 2870(aliphatic $\mathrm{CH}), 1660(\mathrm{C}=\mathrm{O})$, 1640(CONH), 1620(deform. $\left.\mathrm{NH}_{2}\right), \quad 1580(\mathrm{C}=\mathrm{N})$, 1480(deform. $\mathrm{CH}_{3}$ ), 860, 810(aromatic ring).

${ }^{1} \mathrm{H}$ NMR (400 MHz, DMSO- $\left.d_{6}\right) \delta$ (ppm): 14.10(s, $1 \mathrm{H}$, NH of 1,2,4-triazinone), 10.80(s, 1H, NHCO), 8.7(1H, $\mathrm{NH}), 7.55-7.41\left(\mathrm{~m}, 4 \mathrm{H}\right.$, aromatic), 3.49(s, $\left.2 \mathrm{H}, \mathrm{NH}_{2}\right)$, 1.11, 1.00, 0.95(each s, $3 \mathrm{H}, 3 \mathrm{CH}_{3}$ ).

${ }^{13} \mathrm{C}$ NMR (100 MHz, DMSO- $\left.d_{6}\right) \quad \delta(\mathrm{ppm}): \quad 160,152$ $(2 \mathrm{C}=\mathrm{O}), \quad 142(\mathrm{C}=\mathrm{N}), \quad 139(\mathrm{C}-\mathrm{N}), \quad 130-128$ (aromatic carbons), 19.10, 18.80, 18.77(aliphatic carbons). Calculated, $\mathrm{C}_{14} \mathrm{H}_{18} \mathrm{~N}_{6} \mathrm{O}_{2}\left(\mathrm{M}^{+} 302\right)$, \%: C, 55.62; H, 6.00; N, 27.80. Found, \%: C, 55.51; H, 5.89; N, 27.76.

$\mathrm{N}$-[2-(3-(5-(4-Nitrophenyl)-3-thioxo-2,3-dihydro-1H1,2,4-triazol-1-yl)-5-oxo-2,5-dihydro-1,2,4-triazin-6yl)phenyl]pivalamide (4)
A mixture of compound $3(1.50 \mathrm{~g}, 4.96 \mathrm{mmol})$ and 4-nitrobenzoyl isothiocyanate $(1.028 \mathrm{~g}, 4.96 \mathrm{mmol})$ in THF $(20 \mathrm{ml})$ was heated under reflux for $6-8 \mathrm{~h}$. The reaction mixture was cooled to the room temperature. The solid produced was filtered off and crystallized from dioxane to give $\mathbf{4}$ as

orange crystals. Yield 1.539 g, $63 \%$, m.p: $328-330^{\circ} \mathrm{C}$. FT-IR (ATR, $\left.\bar{v}, \mathrm{~cm}^{-1}\right)$ : 3200, 3180(NH, NH), 3060(ArH), 2980, 2890 (aliphatic $\mathrm{CH}$ ), 1665(C=O), 1610(CONH), 1580(C=N), 1560, 1380(asym, \& sym. $\left.\mathrm{NO}_{2}\right), \quad 1550(\mathrm{NCSN}), \quad 1190(\mathrm{C}=\mathrm{S}), \quad 910, \quad 870$, 825(aromatic ring).

${ }^{1} \mathrm{H}$ NMR (400 MHz, DMSO- $\left.d_{6}\right) \delta$ (ppm): 14.10, 11.20 , 8.90(each s, 3H, 3NH), 7.90, 7.78(d,d, 2H, aromatic), 7.71-7.66(m, 2H, aromatic), 7.28-7.42(m, 4H, aromatic), 1.20, 1.01, 0.99(each s, $9 \mathrm{H}, 3 \mathrm{CH}_{3}$ ).

${ }^{13} \mathrm{C}$ NMR (100 MHz, DMSO- $\left.d_{6}\right) \delta(\mathrm{ppm}): 182(\mathrm{C}=\mathrm{S})$, $168(\mathrm{C}=\mathrm{O}), \quad 150(\mathrm{C}=\mathrm{O}), \quad 142(\mathrm{C}=\mathrm{N}), \quad 140(\mathrm{C}=\mathrm{N}), 139$ (C-N), 131-127(aromatic carbons), 19.10, 18.88, $18.86\left(3 \mathrm{CH}_{3}\right)$.

Calculated, $\mathrm{C}_{22} \mathrm{H}_{20} \mathrm{~N}_{8} \mathrm{O}_{4} \mathrm{~S}\left(\mathrm{M}^{+} 492\right)$ \%: C, 53.65; H, 4.09; N, 22.75; S, 6.51. Found, \%: C, 53.59; H, 3.99; $\mathrm{N}, 22.59 ; \mathrm{S}, 6.41 . \mathrm{M} / \mathrm{S}$ (Int. \%): 492(M+, 1.05), 271(5.90), 131(13.99), 122(100), 100(25.11), 85(6.95), 57(25.11)

$\mathrm{N}$-[2-(3-(3-(4-Nitrophenyl)-5-thioxo-4,5-dihydro-1H1,2,4-triazol-1-yl)-5-oxo-2,5-dihydro-1,2,4-triazin-6yl)phenyl]pivalamide (5)

A mixture of compound $3(1.50 \mathrm{~g}, 4.96 \mathrm{mmol})$ and 4-nitrobenzoyl isothiocyanate $(1.028 \mathrm{~g}, 4.96 \mathrm{mmol})$ in EtOH $(20 \mathrm{ml})$ with a few drops of piperidine was heated under reflux for $6-8 \mathrm{~h}$. The reaction mixture was cooled to the room temperature. The solid produced was filtered off and crystallized from dioxane to give $\mathbf{5}$ as

deep-yellowish crystals. Yield 1.661 g, 68\%, m.p: $>360^{\circ} \mathrm{C}$

FT-IR (ATR, $\left.\bar{v}, \mathrm{~cm}^{-1}\right): 3300,3250,3180(3 \mathrm{NH})$, 3080(ArH), 2980, 2870(aliphatic $\mathrm{CH}), 1670(\mathrm{C}=\mathrm{O})$, 1650(CONH), 1580, 1540(C=N), 1550, 1350(asym, \& sym. $\left.\mathrm{NO}_{2}\right), 1188(\mathrm{C}=\mathrm{S}), 920,880,835$ (aromatic ring). ${ }^{1} \mathrm{H}$ NMR (400 MHz, DMSO- $\left.d_{6}\right) \delta$ (ppm): 12.33, 11.20 , 8.88(each s, $3 \mathrm{H}, 3 \mathrm{NH}), 7.88,7.82(\mathrm{~d}, \mathrm{~d}, 2 \mathrm{H}$, aromatic), $7.68-7.59(\mathrm{~m}, \quad 2 \mathrm{H}, \quad$ aromatic $), \quad 7.55-7.40(\mathrm{~m}, \quad 4 \mathrm{H}$, aromatic), 1.11, 1.01, 0.98(each s, 9H, $3 \mathrm{CH}_{3}$ ).

${ }^{13} \mathrm{C}$ NMR (100 MHz, DMSO- $\left.d_{6}\right) \delta(\mathrm{ppm}): 180(\mathrm{C}=\mathrm{S})$, $162(\mathrm{C}=\mathrm{O}), 150(\mathrm{C}=\mathrm{O}), 142(\mathrm{C}=\mathrm{N}), 140(\mathrm{C}=\mathrm{N}), 132$ 127(aromatic carbons), 18.88, 18.20, 18.00 $\left(3 \mathrm{CH}_{3}\right)$.

Calculated, $\mathrm{C}_{22} \mathrm{H}_{20} \mathrm{~N}_{8} \mathrm{O}_{4} \mathrm{~S}\left(\mathrm{M}^{+} 492\right)$ \%: C, 53.65; H, 4.09; N, 22.75; S, 6.51. Found, \%: C, 53.55; H, 3.98; $\mathrm{N}, 22.55 ; \mathrm{S}, 6.11 . \mathrm{M} / \mathrm{S}$ (Int. \%): 492(M+, 1.01), 
192(7.15), 122(100), 103(81.11), 85(3.80), 57(38.11), 55(11.55).

\section{$\mathrm{N}$-[2-(5-Oxo-3-(2-(phenylcarbamothioyl)hydrazino) -} 2,5-dihydro-1,2,4-triazin-6-yl)phenyl]pivalamide (6)

A mixture of compound $3(2.50 \mathrm{~g}, 8.27 \mathrm{mmol})$ and $N$ phenylthiourea $(1.258 \mathrm{~g}, 8.27 \mathrm{mmol})$ in EtOH $(30 \mathrm{ml})$ was heated under reflux for $4 \mathrm{~h}$. The reaction mixture was cooled to the room temperature. The solid produced was filtered off and crystallized from dioxane to give $\mathbf{6}$ as

yellow crystals. Yield 2.025 g, $56 \%$, m.p: $366-368^{\circ} \mathrm{C}$. FT-IR (ATR, $\left.\bar{v}, \mathrm{~cm}^{-1}\right): 3300,3210,3150,3100(\mathrm{NH})$, 3060(ArH), 2970, 2880(aliphatic $\mathrm{CH}), 1680(\mathrm{C}=\mathrm{O})$, $1660(\mathrm{CONH}), 1600,1580(\mathrm{C}=\mathrm{N}), 1188(\mathrm{C}=\mathrm{S}), 920$, 880, 840, 810(aromatic ring).

${ }^{1} \mathrm{H}$ NMR (400 MHz, DMSO- $\left.d_{6}\right) \delta$ (ppm): 12.85(s, $1 \mathrm{H}$, $\mathrm{NH}), 10.55(\mathrm{~s}, 1 \mathrm{H}, \mathrm{CONH}), 8.80(\mathrm{~s}, 1 \mathrm{H}, \mathrm{NH}), 7.83-$ 7.77(m, 4H, aromatic), 7.74-742(m, 5H, Ph), 1.12, $1.08,0.99$ (each s, $3 \mathrm{CH}_{3}$ ).

${ }^{13} \mathrm{C}$ NMR (100 MHz, DMSO- $\left.d_{6}\right) \delta(\mathrm{ppm}): 180(\mathrm{C}=\mathrm{S})$, $162(\mathrm{C}=\mathrm{O}), \quad 154(\mathrm{C}=\mathrm{O}), \quad 142(\mathrm{C}=\mathrm{N}), 139(\mathrm{C}-\mathrm{N}), 132-$ 126(aromatic carbons), 19.11, 18.88, 18.51(3 $\left.\mathrm{CH}_{3}\right)$. Calculated, $\mathrm{C}_{21} \mathrm{H}_{23} \mathrm{~N}_{7} \mathrm{O}_{2} \mathrm{~S}\left(\mathrm{M}^{+}\right.$437), \%: C, 57.65; H, 5.30; N, 22.41; S, 7.33. Found, \%: C, 57.60; H, 5.11; N, 22.12; S, 7.25.

\section{$N-[2-(5-O x o-3-(5-o x o-4-p h e n y l-3-t h i o x o-1,2,4-$ triazolidin-1-yl)-2,5-dihydro-1,2,4-triazin-6-yl) phenyl]pivalamide (7)}

A mixture of $6(1.50 \mathrm{~g}, 3.43 \mathrm{mmol})$ and diethyl carbonate $(0.405 \mathrm{~g}, 3.43 \mathrm{mmol})$ in THF $(20 \mathrm{ml})$ was heated under reflux for $4 \mathrm{~h}$. The reaction mixture was cooled to the room temperature. The solid produced was filtered off and crystallized from dioxane to give 7 as

deep-yellow crystals. Yield 0.794 g, 50\%, m.p: $349-350^{\circ} \mathrm{C}$.

FT-IR (ATR, $\left.\bar{v}, \mathrm{~cm}^{-1}\right): 3300,3180,3150(\mathrm{NH})$, 3080(ArH), 2980, 2870(aliphatic $\mathrm{CH}), 1670(\mathrm{C}=\mathrm{O})$, $1660(\mathrm{C}=\mathrm{O}), 1620(\mathrm{CONH}), 1590(\mathrm{C}=\mathrm{N}), 1330($ cyclic NCSN), 1195(C=S), 880, 840, 810, 790(aromatic ring). ${ }^{1} \mathrm{H}$ NMR (400 MHz, DMSO- $\left.d_{6}\right) \delta(\mathrm{ppm}): 12.50$, 11.30(each s, 2H, 2NH), 7.21-6.99(m, benzo), 1.01, 0.99, 0.89(each s, 3CH 3 ).

${ }^{13} \mathrm{C}$ NMR (100 MHz, DMSO- $\left.d_{6}\right) \delta(\mathrm{ppm}): 188(\mathrm{C}=\mathrm{S})$, $168,162,154(3 \mathrm{C}=\mathrm{O}), 142(\mathrm{C}=\mathrm{N}), 131-128($ aromatic carbons), 119, 116(C5, C6 of 1,2,4-trizine), 20.10 19.01, 18.95, 18.88(aliphatic carbons).

Calculated, $\mathrm{C}_{22} \mathrm{H}_{21} \mathrm{~N}_{7} \mathrm{O}_{3} \mathrm{~S}\left(\mathrm{M}^{+}\right.$463), \%: C, 57.01; H, 4.57; N, 21.15; S, 6.92. Found, \%: C, 56.98; H, 4.42; N, 21.09; S, 6.80 .

\section{$\mathrm{N}$-[2-(3-Amino-4,8-dioxo-1,8-dihydro-4H-[1,2,4]} triazino[4,3-b][1,2,4]triazin-7-yl) phenyl]pivalamide (8)

A mixture of compound 3 (1.50 g, $4.96 \mathrm{mmol})$ and cyanoacetic acid $(0.421 \mathrm{~g}, 4.96 \mathrm{mmol})$ in absolute EtOH $(25 \mathrm{ml})$ with drops of piperidine was heated under reflux for $8 \mathrm{~h}$. The reaction mixture was cooled to the room temperature. The solid produced was filtered off and crystallized from dioxane to give $\mathbf{8}$. Yield 1.304 g, $74 \%$, m.p: $278-280^{\circ} \mathrm{C}$.
FT-IR (ATR, $\left.\bar{v}, \mathrm{~cm}^{-1}\right)$ : 3180(NH), 3080( $\left.\mathrm{NH}_{2}\right), 1688$, $1670(2 \mathrm{C}=\mathrm{O}), \quad 1590(\mathrm{C}=\mathrm{N}), \quad 1470, \quad 1441($ deformation $\mathrm{Me}$ ), 860(substituted $\mathrm{Ph}$ ).

${ }^{1} \mathrm{H}$ NMR (400 MHz, DMSO- $\left.d_{6}\right) \delta$ (ppm): 11.2, $8.99(\mathrm{NH}), 7.88-7.55(\mathrm{~m}, 4 \mathrm{H}$, aromatic protons $), 3.45(\mathrm{~s}$, $2 \mathrm{H} \mathrm{NH}_{2}$ ), 1.01, 0.98, 0.90(each s, $3 \mathrm{CH}_{3}$ ).

Calculated, $\mathrm{C}_{16} \mathrm{H}_{17} \mathrm{~N}_{7} \mathrm{O}_{3}\left(\mathrm{M}^{+} 355\right)$ \%: C, 54.08; $\mathrm{H}, 4.82 ; \mathrm{N}, 27.59$. Found, \%: C, 53.91; H, 4.66; $\mathrm{N}, 27.40$.

\section{$\mathrm{N}$-[2-(4-Amino-8-oxo-1,8-dihydro-2H-[1,2,4]} triazino[4,3-b][1,2,4]triazin-7-yl) phenyl]pivalamide (9)

A mixture of compound $3(1.50 \mathrm{~g}, 4.96 \mathrm{mmol})$ and 2-chloroacetonitrile $(0.372 \mathrm{~g}, 4.96 \mathrm{mmol})$ in DMF $(25 \mathrm{ml})$ was heated under reflux for $4 \mathrm{~h}$. The reaction mixture was cooled to the room temperature, then poured onto ice. The solid produced was filtered off and crystallized from $\mathrm{EtOH}$ to give 9.

Yield 1.168 g, $69 \%$, m.p: $288-290^{\circ} \mathrm{C}$.

FT-IR (ATR, $\left.\bar{v}, \mathrm{~cm}^{-1}\right): 3300,3250,3150\left(\mathrm{NH}_{2}, \mathrm{NH}\right)$, $1680, \quad(\mathrm{C}=\mathrm{O}), \quad 1650(\mathrm{CONH}), \quad 1630\left(\mathrm{NH}_{2}\right), \quad 1480$, 1440(deformation Me), 860(substituted $\mathrm{Ph}$ ).

${ }^{1} \mathrm{H}$ NMR (400 MHz, DMSO- $\left.d_{6}\right) \delta$ (ppm): 12.20, 11.11, 9.5(each s, $3 \mathrm{NH}), 7.88-7.51(\mathrm{~m}, 4 \mathrm{H}$, aromatic protons), 3.51(s, $2 \mathrm{H} \mathrm{NH}_{2}$ ), 1.01, 0.98, 0.95(each s, $3 \mathrm{CH}_{3}$ ).

Calculated, $\mathrm{C}_{16} \mathrm{H}_{19} \mathrm{~N}_{7} \mathrm{O}_{2}\left(\mathrm{M}^{+} 341\right)$ \%: $\mathrm{C}, 56.29$; $\mathrm{H}, 5.61 ; \mathrm{N}, 28.72$. Found, \%: C, 55.95; H, 5.47; N, 28.67 .

\section{$\mathrm{N}$-[2-(4-imino-8-oxo-3-phenyl-1,8-dihydro-4H- [1,2,4]triazino[4,3-b][1,2,4]triazin-7-yl)phenyl] pivalamide (10)}

A mixture of compound $3(1.50 \mathrm{~g}, 4.96 \mathrm{mmol})$ and $(0.650 \mathrm{~g}, 4.96 \mathrm{mmol})$ in EtOH $(25 \mathrm{ml})$ with drops of piperidine was heated under reflux for $8 \mathrm{~h}$. The reaction mixture was cooled to the room temperature, then added dil. $\mathrm{HCl}$. The solid produced was filtered off and crystallized from EtOH to give $\mathbf{9}$.

Yield 1.319 g, $64 \%$, M.p: $170-272^{\circ} \mathrm{C}$.

FT-IR (ATR, $\left.\quad \bar{v}, \quad \mathrm{~cm}^{-1}\right): \quad 3400(\mathrm{OH}), \quad 3150(\mathrm{NH})$, 3080(NHCO), 1680, $(\mathrm{C}=\mathrm{O}), 1660(\mathrm{C}=\mathrm{O}), 1580(\mathrm{C}=\mathrm{N})$, 1480, 1440 (deformation Me), 880(substituted $\mathrm{Ph}$ ).

${ }^{1} \mathrm{H}$ NMR (400 MHz, DMSO- $\left.d_{6}\right) \delta$ (ppm): 11.11, 10.50(each s, NH, NHCO), 8.90(OH), 7.80-7.66 (m, 4H, aromatic protons), 1.01, 0.90, 0.88(each s, $\left.3 \mathrm{CH}_{3}\right)$.

Calculated, $\mathrm{C}_{22} \mathrm{H}_{21} \mathrm{~N}_{7} \mathrm{O}_{2} \quad\left(\mathrm{M}^{+}\right.$415), \%: C, 63.60; H, 5.10; N, 23.60. Found, \%: C, 63.49; H, 5.02; $\mathrm{N}, 23.37$.

\section{The in vitro antibacterial evaluation}

The new synthesized systems were investigated as in vitro antibacterial agents such as Gram-positive bacteria involved Bacillus subtilis, Bacillus cereus, Sarcina lutea, and Micrococcus luteus; and Gramnegative bacteria involved Pseudomonas aeruginosa, Escherichia coli, Acinetobacter johnsonii, and Xanthomonas oryzae, by using the conventional well/disc. ${ }^{3}$ Tetracycline $(30 \mu \mathrm{g} /$ dis., $6 \mathrm{~mm}$ in diameter) used as a control. 
The new compounds were dissolved in 5\% DMSO to obtain a $0.5 \%$ stock solution. Grown cultures were used sterile nutrient agar medium in each Petri plate. The applied concentration in each 10, 30, and $50 \mathrm{~mL}$ of stock solution added. All the plates were incubated at $28^{\circ} \mathrm{C}$ for $24 \mathrm{~h}$ and the size of the resulted zone of inhibition determined.

\section{Conflict of interest}

The authors declare no conflicts of interest.

\section{References}

1- R. M. Abdel-Rahman, M. S. T. Makki, Abeer N. Al-Romaizan, Synthesis of novel fluorine substituted isolated and fused heterobicyclic nitrogen systems bearing 6-(2'-phosphorylanilido)-1,2,4-triazin-5-one moiety as a potential inhibitor towards HIV-1 activity. International Journal of Organic Chemistry, 2014, 4(04), 247-268.

2- S. Cascioferro, B. Parrino, V. Spanò, A. Carbone, A. Montalbano, P. Barraja, P. Diana, G. Cirrincione, An overview of the recent developments of 1,2,4-triazine derivatives as anticancer compounds. European Journal of Medicinal Chemistry, 2017, 142, 328-375.

3- W.A. Bawazir, R.M. Abdel-Rahman, Synthesis of new fluorinated amino-heterocyclic compounds bearing 6-aryl-5-oxo-1,2,4-triazin-

3 -yl moiety as antimicrobial agents. International Journal of Organic Chemistry, 2018, 8(4), 349-358.

4- R. M. Abdel-Rahman, W. A. Bawazir, Various routes to synthesis 3-thioxo-1,2,4-triazine-5-one derivatives as antimicrobial agents. International Journal of Organic Chemistry, 2018, 8(2), 191-200.

5- R. M. Abdel-Rahman, M. S. T. Makki, W. A. Bawazir, Synthesis of some more fluorine heterocyclic nitrogen systems derived from sulfa drugs as photochemical probe agents for inhibition of vitiligo disease-part i. Journal of Chemistry, 2011, 8(1), 405-414.

6- M. S. T. Makki, R. M. Abdel-Rahman, Abdulrahman S. Alharbi, Synthesis and antiinflammatory effect of some more new fluorinated 3-substituted amino/ 3,5-diamino1,2,4-triazine derivatives as Lamotrigine analogs. Current Organic Synthesis, 2019, 16 (1),
165-172.

7- A. N. Al-Romaizan, M. S. T. Makki, R. M. Abdel-Rahman, Synthesis of new fluorine/phosphorus substituted 6-(2'-aminophenyl)-3-thioxo-1,2,4-triazin-5(2H,4H) one and their related alkylated systems as molluscicidal agent as against the snails responsible for Bilharziasis diseases. International Journal of Organic Chemistry, 2014, 4(2), 154-168.

8- M. S. T. Makki, R. M. Abdel-Rahman, Abdulrahman S. Alharbi, Synthetic approach for novel fluorine substituted $\alpha$-amino-phosphonic acids containing 1,2,4-triazin-5-one moiety as antioxidant agents. International Journal of Organic Chemistry, 2018, 8(1), 1-15.

9- D. A. Bakhotmah, R. M. Abdel-Rahman, A Review on the Synthesis and Chemistry of Bioactive Pyrazolines Bearing 1,2,4-Triazine Moieties. Mini-Reviews in Organic Chemistry, 2016, 13 (1), 62-77.

10- F. M. S. Aqlan, M. S. T. Makki, R. M. AbdelRahman, Synthesis, Spectroscopic Studies of Fluorinated Pyrimido-1,2,4-Triazines: Protective Effect Against Some Plant Pathogenic Fungi. Journal of Heterocyclic Chemistry, 2016, 53 (4), 1310-1317.

11- R. M. Abdel-Rahman, W. R. Abdel-Monem, Chemical reactivity of 3-hydrazino-5,6-diphenyl1,2,4-triazine towards $\pi$-acceptors activated carbonitriles. Indian Journal of Chemistry, 2007, 46B, 838-846.

12- R. M. Abdel-Rahman, Reaction of 3-hydrazino5,6-diphenyl-1,2,4-triazine with unsymmetrical 1,3-bycarbonyl compounds-synthesis of some new 3-(3',5'-disubstituted pyrazol-1'-yl)-5,6diphenyl-1,2,4-triazines and their antimicrobial activity. Indian Journal of Chemistry Section B-Organic chemistry including medicinal chemistry, 1988, 27(6), 548-553.

13- D. A. Bakhotmah, R. M. Abdel-Rahman, Synthesis and structural determination of novel fluorinated steroidal spiro(pyrazolo[4,3-e] $[1,2,4]$ triazin-3'-yl) derivatives as affecting enzymatic agents. Letters in Organic Chemistry, 2017, 14(2), 134-140.

14- R. M. Abdel-Rahman, Synthesis and anti-human immune virus activity of some new fluorinecontaining substituted-3-thioxo-1, 2, 4-triazin-5ones. Farmaco, 1991, 46 (2), 379-389. 